\title{
DNA copy number analysis of metastatic urothelial carcinoma with comparison to primary tumors
}

\author{
Richard M Bambury ${ }^{1 *+}$, Ami S Bhatt ${ }^{2,3+}$, Markus Riester ${ }^{2}$, Chandra Sekhar Pedamallu ${ }^{2,3}$, Fujiko Duke ${ }^{2,3}$, \\ Joaquim Bellmunt ${ }^{2}$, Edward C Stack², Lillian Werner², Rachel Park², Gopa lyer ${ }^{1}$, Massimo Loda ${ }^{2,3}$, Philip W Kantoff ${ }^{2}$, \\ Franziska Michor ${ }^{2}$, Matthew Meyerson ${ }^{2,3}$ and Jonathan E Rosenberg ${ }^{1}$
}

\begin{abstract}
Background: To date, there have been no reports characterizing the genome-wide somatic DNA chromosomal copy-number alteration landscape in metastatic urothelial carcinoma. We sought to characterize the DNA copynumber profile in a cohort of metastatic samples and compare them to a cohort of primary urothelial carcinoma samples in order to identify changes that are associated with progression from primary to metastatic disease.

Methods: Using molecular inversion probe array analysis we compared genome-wide chromosomal copynumber alterations between 30 metastatic and 29 primary UC samples. Whole transcriptome RNA-Seq analysis was also performed in primary and matched metastatic samples which was available for 9 patients.

Results: Based on a focused analysis of 32 genes in which alterations may be clinically actionable, there were significantly more amplifications/deletions in metastases ( $8.6 \%$ vs $4.5 \%, \mathrm{p}<0.001)$. In particular, there was a higher frequency of E2F3 amplification in metastases (30\% vs 7\%, $p=0.046)$. Paired primary and metastatic tissue was available for 11 patients and 3 of these had amplifications of potential clinical relevance in metastases that were not in the primary tumor including ERBB2, CDK4, CCND1, E2F3, and AKT1. The transcriptional activity of these amplifications was supported by RNA expression data.
\end{abstract}

Conclusions: The discordance in alterations between primary and metastatic tissue may be of clinical relevance in the era of genomically directed precision cancer medicine.

\section{Background}

Bladder cancer is diagnosed in approximately 400,000 people and causes 150,000 deaths worldwide each year [1]. The majority of urinary tract cancers in the developed world are of urothelial carcinoma (UC) histology [2]. Extensive data characterizing the genetic profile of primary UC has been published and includes The Cancer Genome Atlas (TCGA) project which comprehensively describes the molecular features of primary muscle-invasive bladder UC [3]. These studies have identified several recurrent and therapeutically targetable genetic alterations but have focused on primary tumor characterization rather than the

\footnotetext{
* Correspondence: bamburyr@mskcc.org

${ }^{\dagger}$ Equal contributors

${ }^{1}$ Memorial Sloan Kettering Cancer Center/Weill Cornell Medical College, New York, USA

Full list of author information is available at the end of the article
}

metastatic lesions that ultimately cause patient death. In muscle-invasive UC, these alterations include somatic point mutations in TP53 (35-50\%), PIK3CA (15-20\%) and FGFR3 (10-15\%) [3-5]. Inactivating mutations commonly occur in chromatin remodeling genes, most frequently MLL2, ARID1A and KDM6A, each of which occur in approximately $25 \%$ of cases $[3,6]$. Furthermore, oncogenic somatic copy-number alterations (SCNAs) have been described including deletion of $R B 1$ in 14-15\% and amplification of $E R B B 2$ in $5-7 \%$ of cases [3,5]. Copy number loss in chromosome 9 and copy number gain in the q arm of chromosome 8 are common, although their exact biologic significance is uncertain $[7,8]$. Previous studies have shown that FGFR3 and KDM6A mutations are associated with lower grade and stage primary tumors, while $R B 1$ deletion and TP53 mutations are more common in high-grade 
tumors [4,6-8]. One study used next-generation sequencing to examine alterations in 182 cancer-related genes in a cohort of 35 locally advanced or metastatic UC patients [9]. The majority of samples analyzed were from the primary tumor and results were broadly similar to what was previously reported in muscle-invasive primary bladder UC cohorts [9]. In this study, we sought to characterize the genome-wide SCNA profile in a cohort of metastatic UC samples. Furthermore, we compared these metastases to primary tumors using SCNA and RNA expression analysis to understand the genetic and transcriptomic differences between these two disease states and to identify changes associated with progression from primary to metastatic disease.

\section{Methods}

Details on the site of tumor tissue, normal tissue, age and gender for the 46 individual cases analyzed are outlined in Table 1 and Additional file 1: Table S1. 30 metastatic UC samples were analyzed from lung, node and other sites. These were compared with 29 primary UC samples mostly of bladder origin. Paired primary and metastatic tissue was available for the same patient in 11 cases. RNA data was available in 9 of these 11 matched pairs.

Following pathologic examination, tumor DNA was extracted from formalin fixed paraffin embedded (FFPE) tissue using the QIAamp DNA FFPE Tissue Kit (Qiagen, Valencia, CA) as previously described [10]. Where available, normal DNA for comparison was extracted from adjacent histopathologically normal lymph nodes, renal parenchyma, seminal vesicle, prostate or lung tissue. Using the same samples, total RNA was extracted when possible using the automated Beckman Coulter Biomek FxP platform and the Agencourt Formapure Kit.

Copy number analysis for normal, primary tumor and metastatic DNA was performed using MIP array technology (Affymetrix OncoScan FFPE Express 2.0) with 334,183 sequence tag site probes which were used to measure DNA copy number at different loci across the

Table 1 Sites of primary tumour and metastases analysed

\begin{tabular}{lll}
\hline & $\mathbf{n}$ & \% \\
\hline Primary tumors $(\mathbf{n}=\mathbf{2 9})$ & 24 & $83 \%$ \\
Bladder & 5 & $17 \%$ \\
Upper tract & & \\
Metastases $(\mathbf{n}=\mathbf{3 0})$ & 10 & $33 \%$ \\
Lung & 6 & $20 \%$ \\
Peritoneum & 6 & $20 \%$ \\
Lymph node & 3 & $10 \%$ \\
Brain & 5 & $17 \%$ \\
Other & 5
\end{tabular}

Details of individual cases are outlined in Additional file 1: Table S1. human genome [11]. Probes were spaced at a median of $9 \mathrm{~kb}$ between each locus but were distributed closer together at known oncogenes and tumor suppressor genes. Copy number data were processed and normalized by Affymetrix as previously described [11]. Copy numbers were estimated with the NEXUS software and only samples that passed Affymetrix quality control metrics (median absolute pairwise difference $[\mathrm{MAPD}]$ value of $\leq 0.6$ ) were considered [12].

Two micrograms of total RNA from each sample was utilized for sequencing library construction. Complementary DNA (cDNA) synthesis and bar-coded sequencing library preparation was performed as previously described $[13,14]$ with the following modifications: Double-stranded cDNA synthesis was performed using random hexamers and cDNA was purified using QiagenTM mini-elute columns. Samples were mixed (six samples per lane of Illumina V3 HiSeq sequencing) and 101 base pair pairedend sequencing was performed. The resultant data was aligned to the human reference genome (hg19) and exon-exon junctions (ensembl v64) with the PRADA pipeline [15]. Non-human sequences were taxonomically characterized using PathSeq, as previously described [16]. Gene-level expression values [in reads per kilobase per million mapped reads (RPKM)] were generated by RNA-Seq for transcriptomic analysis [17].

The frequency of SCNA across the whole genome was assessed to compare alteration frequencies between primary tumors and metastases. A focused analysis was also performed to look for amplifications/deletions in genes involved in proliferation and cell-cycle control known to commonly harbor oncogenic alterations in UC and for which targeted therapies are currently under investigation $[3,5]$. This focused analysis also examined the frequency of amplifications/deletions in regions found to have statistically significant focal SCNAs using the Genomic Identification of Significant Targets in Cancer version 2.0 algorithm (GISTIC2.0) in the TCGA analysis [3].

There are no standardised $\log _{2}$ ratio cut-offs to define low-amplitude copy number gain/loss and high amplitude amplification/deletion. Based on the available published literature, we used a $\log _{2}$ ratio cut-off of $+/-0.25$ to define copy number gain/loss and a $\log _{2}$ ratio cut-off of $+/-$ 0.8 to define amplification and deletion $[7,18,19]$.

Normalized copy number data was segmented using GLAD with default parameters available in GenePattern version 3.3.3 [20]. GISTIC 2.0 (v2.0.12) was then used to identify regions of the genome that were significantly gained or deleted across a set of samples using a Q-value cutoff $<0.25$ [21]. This algorithm is designed to identify significant driver SCNAs in human cancers by taking into account the frequency and amplitude of the SCNA and comparing it to the background rate of SCNAs across the genome. The algorithm 
Table 2 Frequency of amplifications and deletions in a focused analysis of 32 genomic regions which were either previously known to be of interest in urothelial cancer or which were identified by TCGA as having statistically significant focal copy number changes

\begin{tabular}{|c|c|c|c|}
\hline Pathway & Gene & $\%$ alterations in primaries $(n=29)$ & $\%$ alterations in metastasis $(n=30)$ \\
\hline \multirow[t]{11}{*}{ MAP kinase pathway } & $E R B B 2 \wedge$ & $2 / 29-7 \%$ & $4 / 30-13 \%$ \\
\hline & FGFR3^ & 0/29-0\% & $1 / 30-3 \%$ \\
\hline & $F G F R 1 \wedge$ & $2 / 29-7 \%$ & $4 / 30-13 \%$ \\
\hline & $E G F R \wedge$ & 0/29-0\% & $0 / 30-0 \%$ \\
\hline & MET^ & 0/29-0\% & 0/30-0\% \\
\hline & KRAS^ & 0/29-0\% & $0 / 30-0 \%$ \\
\hline & $N F 1 \wedge$ & 0/29-0\% & $1 / 30-3 \%$ \\
\hline & $B R A F \wedge$ & 0/29-0\% & $0 / 30-0 \%$ \\
\hline & $R A F 1 \wedge$ & $1 / 29-3 \%$ & $3 / 30-10 \%$ \\
\hline & $M Y C \wedge$ & 0/29-0\% & $2 / 30-7 \%$ \\
\hline & $M Y C L 1 \wedge$ & $5 / 29-17 \%$ & $3 / 30-10 \%$ \\
\hline \multirow[t]{2}{*}{ P53 pathway } & $M D M 2 \wedge$ & $1 / 29-3 \%$ & $3 / 30-10 \%$ \\
\hline & $\operatorname{TP} 53^{\mathrm{V}}$ & $2 / 29-7 \%$ & $2 / 30-7 \%$ \\
\hline \multirow[t]{6}{*}{ RB pathway* } & $C D K N 2 A^{\vee}$ & $8 / 29-28 \%$ & $10 / 30-33 \%$ \\
\hline & CDK4^ & $1 / 29-3 \%$ & $2 / 30-7 \%$ \\
\hline & CCND1^ & $2 / 29-7 \%$ & $6 / 30-20 \%$ \\
\hline & CCNE1^ & $2 / 29-7 \%$ & $4 / 30-13 \%$ \\
\hline & $R B 1^{\vee}$ & 0/29-0\% & $2 / 30-7 \%$ \\
\hline & $E 2 F 3 \wedge *(p=0.04)$ & $2 / 29-7 \%$ & $9 / 30-30 \%$ \\
\hline \multirow[t]{5}{*}{ PI3K pathway } & PTEN $^{\vee}$ & $1 / 29-3 \%$ & $1 / 30-3 \%$ \\
\hline & PIK3CA^ & 0/29-0\% & $0 / 30-0 \%$ \\
\hline & $A K T 1 \wedge$ & 0/29-0\% & $1 / 30-3 \%$ \\
\hline & $\mathrm{TSCl}^{\vee}$ & $1 / 29-3 \%$ & $1 / 30-3 \%$ \\
\hline & MTOR^ & 0/29-0\% & 0/30-0\% \\
\hline \multirow[t]{9}{*}{ Others } & $B C L 2 L 1 \wedge$ & $1 / 29-3 \%$ & $1 / 30-3 \%$ \\
\hline & PPARG^ & $1 / 29-3 \%$ & $5 / 30-17 \%$ \\
\hline & $C R E B B P^{\vee}$ & 0/29-0\% & $1 / 30-3 \%$ \\
\hline & $P \vee R L 4 \wedge$ & $2 / 29-7 \%$ & $7 / 30-23 \%$ \\
\hline & YWHAZ^ & $5 / 29-17 \%$ & $4 / 30-13 \%$ \\
\hline & $N C O R 1^{\vee}$ & 2/29-7\% & $1 / 30-3 \%$ \\
\hline & YAP1^ & 0/29-0\% & $1 / 30-3 \%$ \\
\hline & ZNF703^ & $1 / 29-3 \%$ & $3 / 30-10 \%$ \\
\hline & & $n=928$ & $n=960$ \\
\hline$\%$ total loci with amplification/deletion* & $(p<0.001)$ & $42 / 928-4.5 \%$ & $83 / 960-8.6 \%$ \\
\hline
\end{tabular}

*p $<0.05$ Fishers exact test, $\wedge=$ amplification, ${ }^{\vee}=$ deletion.

The data are represented using a threshold of $\log _{2}$ copy number ratio $>0.8$ for amplification and $\log _{2}$ copy number ratio $<-0.8$ for deletion. Data are shown in tabular format with frequency of amplifications and deletions of genes outlined. ^denotes amplifications and "denotes deletions.

compensates for the different background frequencies of SCNAs of varying length and quantifies the likelihood of copy-number alterations being biologically relevant in the form of a q-value. The software estimated false discovery rates (q-values), as well as potential targets (drivers) within the copy number aberrant regions. Threshold for copy number gain and loss was set at $+/-0.25$ so that approximately $99 \%$ of all segments in normal samples were below this threshold. We defined broad alterations as those spanning $>50 \%$ of a chromosome arm.

To infer the relative similarity between the DNA and RNA profiles of normal, primary and metastatic samples, unsupervised hierarchical clustering was 


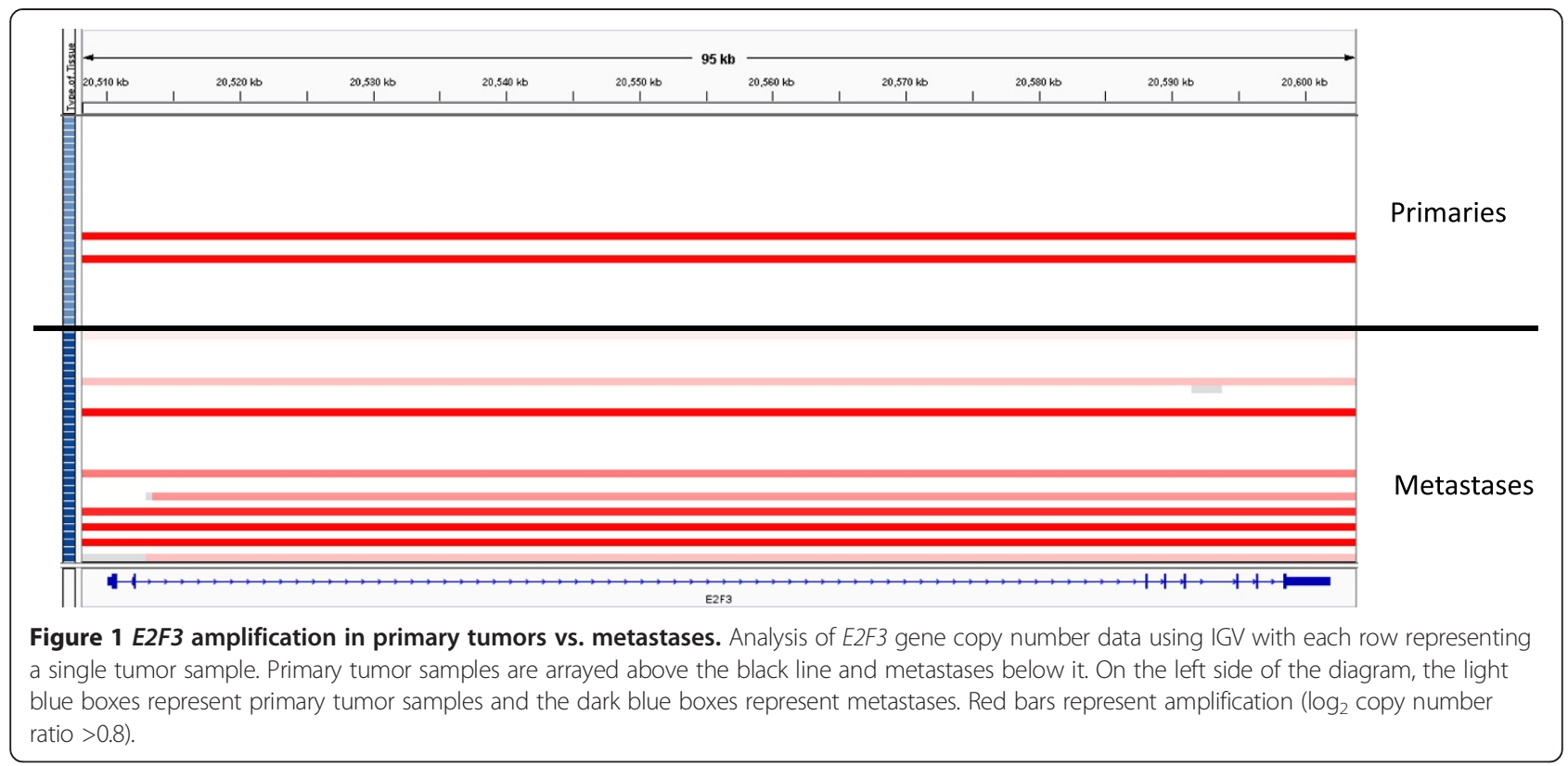

performed as follows: for the DNA data, hierarchical clustering was performed using the pvclust $\mathrm{R}$ package with 1000 bootstrap iterations, Ward's clustering method and otherwise default parameters. The boot strapping procedure estimates how strongly the clusters are supported by data. Bootstrap values are reported as percentages and indicate how often a cluster was observed in the bootstrapping. They are obtained by multiscale $[22,23]$ and by normal resampling, i.e. sampling with replacement.

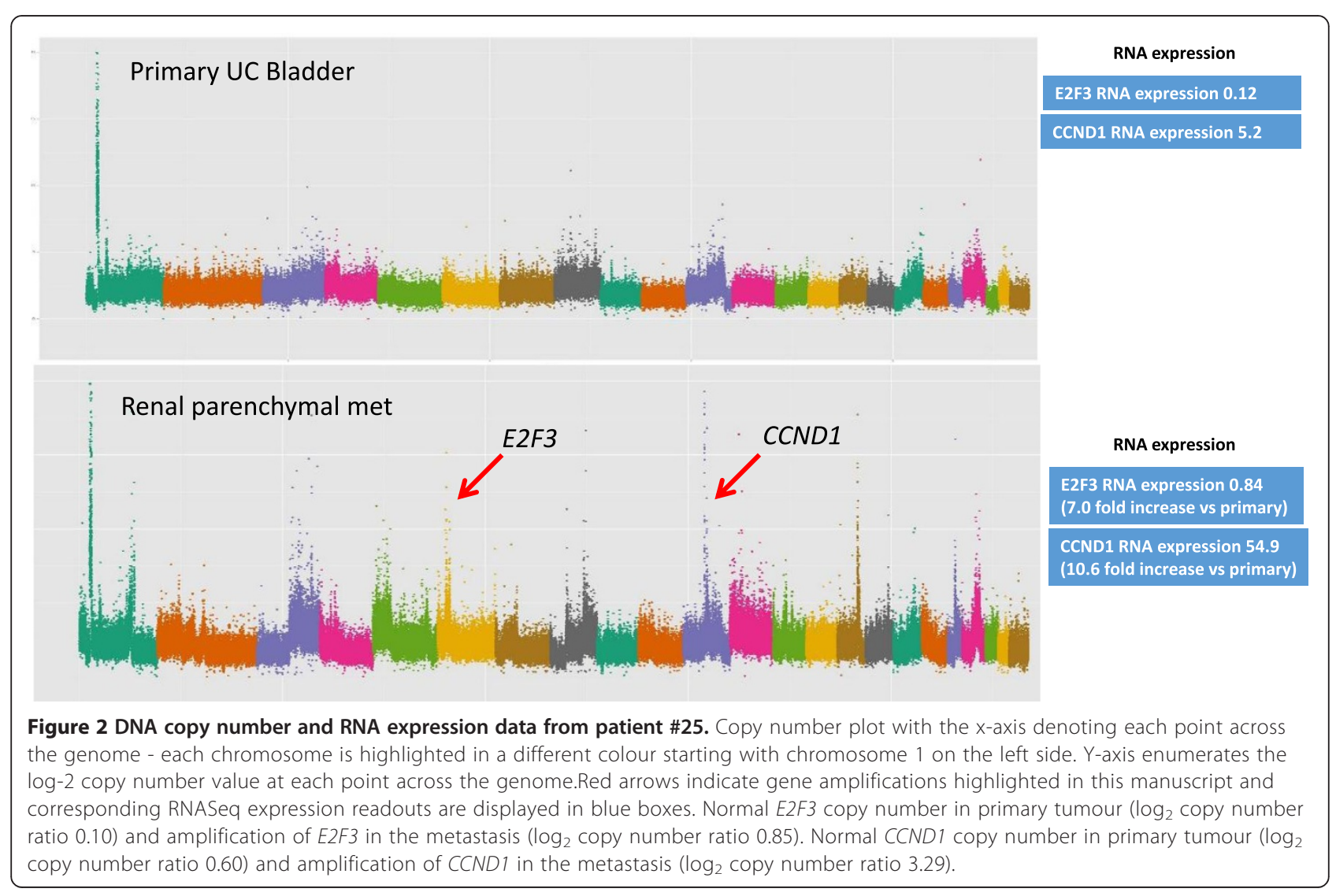


For RNA data, unsupervised hierarchical clustering was performed and RNA-Seq RPKM values were log2 + 1 transformed. Invariantly expressed genes were removed using the genefilter $\mathrm{R}$ package. Using the default settings of this package, we removed $50 \%$ of the genes with lowest interquartile range (IQR). Clustering was then performed with the same parameters we used for the DNA data.

To further test for the clonality of matched primary tumors and metastases, the Clonality testing $\mathrm{R}$ package tool developed at Memorial Sloan Kettering Cancer Center was used to analyze the DNA copy number data [22-24]. This is an $\mathrm{R}$ package for testing whether two tumors from the same patient are clonal (metastasis) or independent (synchronous primaries) based on their genome wide copy number profiles.

For the RNA data, heatmaps and tables of differentially expressed genes in normal bladder vs. primary and metastases and in primary vs. metastases are presented (Additional file 2: Figure S1 and Additional file 3: Figure S2).

All samples were collected under protocols approved by the Institutional Review Board (IRB) at Dana Farber Cancer Institute, de-identified and approved for use by the DFCI IRB.

\section{Results}

Focused analysis of 32 selected genes by Iyer et al. described the prevalence and co-occurrence of potentially actionable alterations in a group of 21 genes from signaling pathways known to be relevant in primary UC bladder [5]. We compared the frequency of amplifications and deletions between primary tumors and metastases in these 21 genes as well as another 11 regions found to have statistically significant focal SCNAs in the TCGA analysis (Table 2) [3-7,9]. A $\log _{2}$ ratio cutoff of $+/-0.8$ was used to stringently define gene amplification and deletion, respectively, based on prior published studies as described in the methods section $[7,18,19]$. Overall, there were more amplifications/deletions in these genes in metastases compared with primary tumors $(8.6 \%$ loci altered vs. $4.5 \%, \mathrm{p}<0.001$ Fishers exact, Table 2). In an individual gene-wise comparison, there were more E2F3 amplifications in metastases compared with primary tumours (30\% vs. $7 \%$, $\mathrm{p}=0.041$ Fishers exact, Table 2 and Figure 1). In 2 of the 11 patients with matched primary and metastatic tissue, E2F3 amplifications were present in the metastasis but not in the matched primary tumor. In these cases, there was associated increased E2F3 RNA

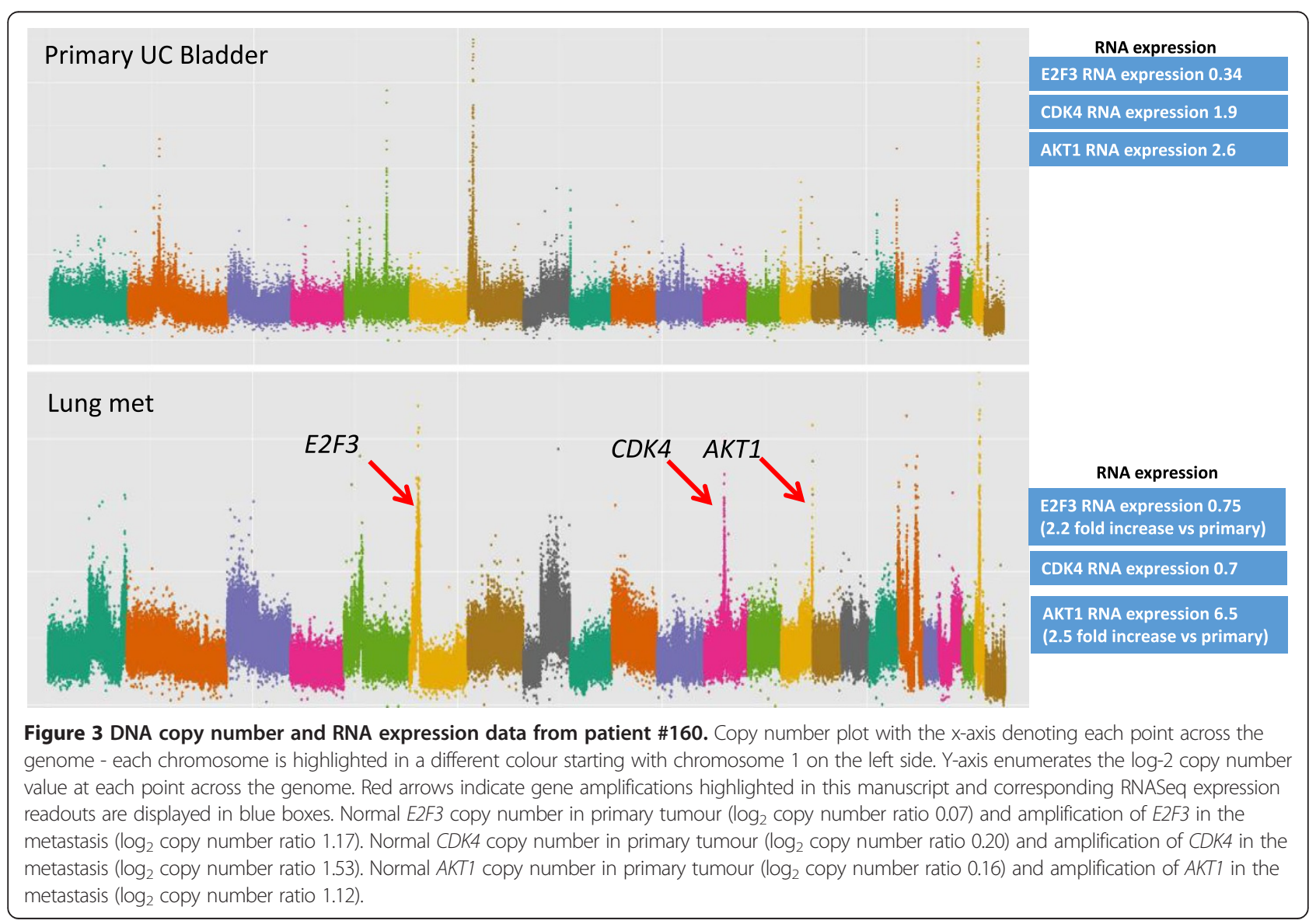


expression in the metastasis compared with the matched primary (patients 25 and 169, Figures 2 and 3). The SOX4 locus is located close to E2F3, is co-amplified in many of these cases, and was identified by TCGA investigators as another potentially biologically relevant gene in this amplicon [3]. Indeed in these two patients (25 and 169) there was a 2-fold increased RNA expression of SOX4 between the primary and metastasis specimens that paralleled the gene amplification.

\section{Instances of discordant genetic alterations between paired primary and metastatic samples}

In 3 of 11 patients for whom primary and metastatic tissue was available, potentially clinically actionable amplifications were observed in metastases but not in the matched primary tumours (Figures 2, 3 and 4). In the first case (patient 25), E2F3 and CCND1 amplifications were detected in a soft tissue renal metastasis and were not present in the corresponding bladder primary (Figure 2). RNA expression data was concordant with these findings with 7.0-fold and 10.6-fold increased expression levels of E2F3 and $C C N D 1$ respectively. The second case (patient 160) had amplifications of $E 2 F 3, C D K 4$ and $A K T 1$ in a lung metastasis which were not present in the bladder primary (Figure 3). RNA expression data confirmed increased E2F3 and AKT1 expression but not increased CDK4 expression. The third case (patient 63) had ERBB2 ampli fication in a lymph node metastasis that was not present in the corresponding bladder primary (Figure 4). RNA expression data was not available for this patient. When examining these 32 genes, we did not find any instance of amplification or deletion in the primary tumor that was not present in a matched metastasis.

\section{Frequency of low-amplitude copy number alterations}

The frequency of low-amplitude SCNAs across the whole genome was compared between primary and metastatic tumors. A $\log _{2}$ ratio cut-off of $+/-0.25$ was used to define low-amplitude SCNAs as described in the methods section. Of note, the limited sample size meant our power to detect significant differences after correcting for multiple testing was only 0.4 . On a genome-wide basis, the overall fraction of altered loci was not significantly different between primary tumors and metastases. There was a trend towards

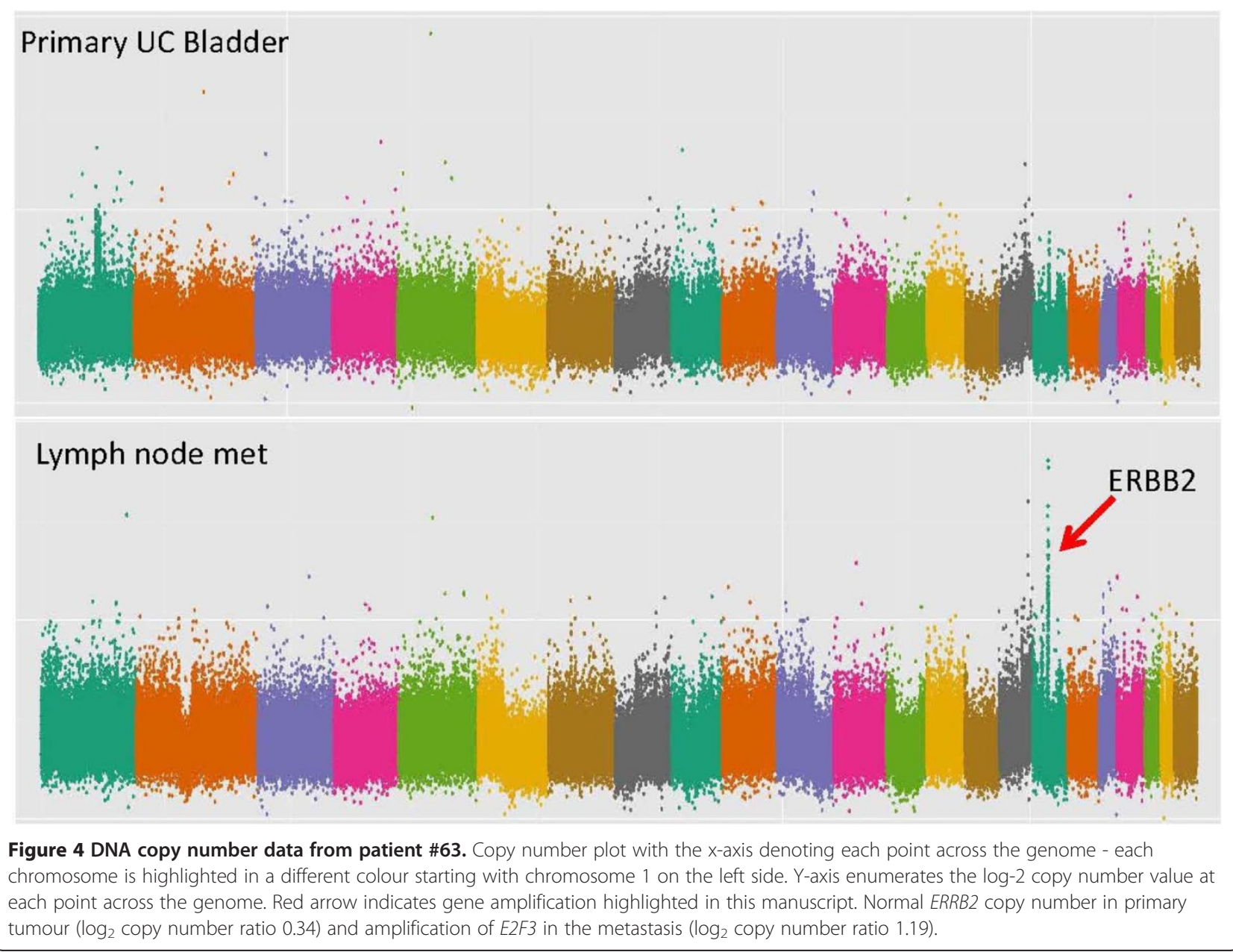


more chromosome 4 CNLs in metastases compared with primary tumors (Figure 5), although the difference was not statistically significant after correction for multiple testing ( $p=0.01$ for chromosome $4 q$ and $p=0.03$ for chromosome $4 \mathrm{p}$ - paired $\mathrm{t}$-test; FDR $=0.31$ ). The trend to more frequent chromosome $4 \mathrm{CNL}$ events in metastases was also observed when the analysis was restricted to paired primary and metastatic tissue specimens from the same patients ( $\mathrm{p}=0.04$ for Chr $4 \mathrm{q}$ and $\mathrm{p}=0.09$ for $4 \mathrm{p}$ - paired $\mathrm{t}$-test) , suggesting that a proportion of tumors may lose genetic material from chromosome 4 when progressing from primary to metastatic disease (Additional file 4: Figure S3). Whether this loss represents a driver or passenger event is unclear.

\section{GISTIC 2.0}

GISTIC 2.0 analysis of the primary and metastatic cohorts (designed to identify significant driver SCNAs) demonstrated multiple regions of significant SCNA as previously described in other cohorts of UC patients $[3,5,7,8,21]$. These include regions of amplification at E2F3, ERBB2 and PPARG and deletion at CDKN2A in both primary (Figure 6a) and metastatic (Figure 6b) cohorts. A recent study, using primary tumour tissue for analysis, reported that amplification at the 1q23.3 locus was associated with worse prognosis in metastatic UC [25]. In GISTIC 2.0 analysis of our dataset, 1q23.3 was found to be significantly amplified in both primary and metastatic samples.

\section{Hierarchical clustering analysis}

Hierarchical cluster analysis using DNA copy number data confirmed that the paired primary and metastatic samples from the same patients cluster together in all cases suggesting clonality (i.e. that they had initially arisen from a single cell of origin) (Figure 7a). These findings were further corroborated using the MSKCC clonality tool which suggested a high likelihood that 10 of the 11 paired primary and metastatic tumors had arisen from the same cell of origin rather than from different primary tumors. For one patient (patient 169), there was a weak and statistically non-significant trend towards independence. (Additional file 5: Figure S4); however, the primary and metastasis samples from this patient clustered together on hierarchical clustering analysis, suggesting clonality (Figure 7a).

Hierarchical clustering analysis using the RNA expression data from normal and tumor tissue found that the normal tissue specimens clustered together and independent of primary and metastatic tumor samples

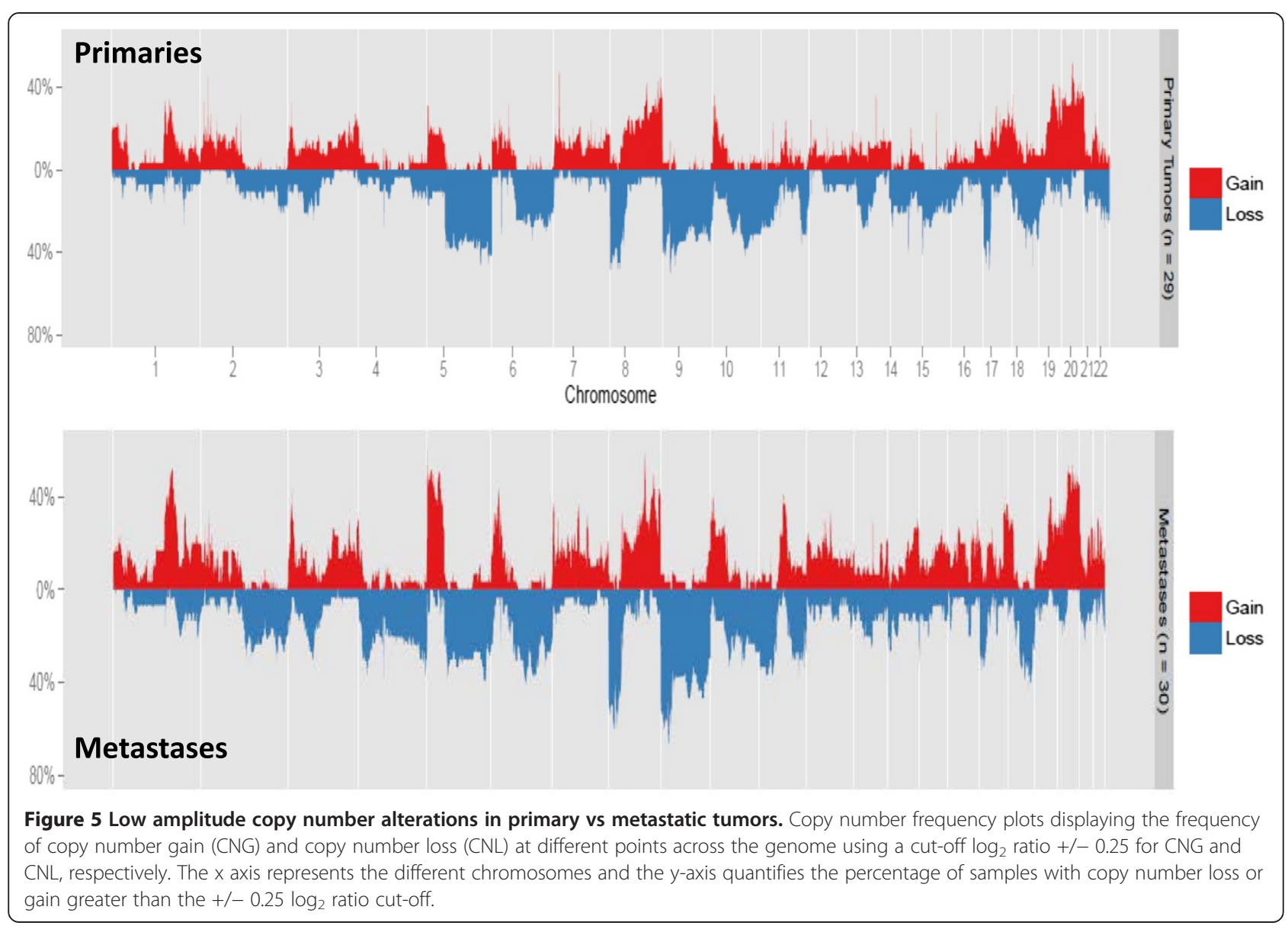




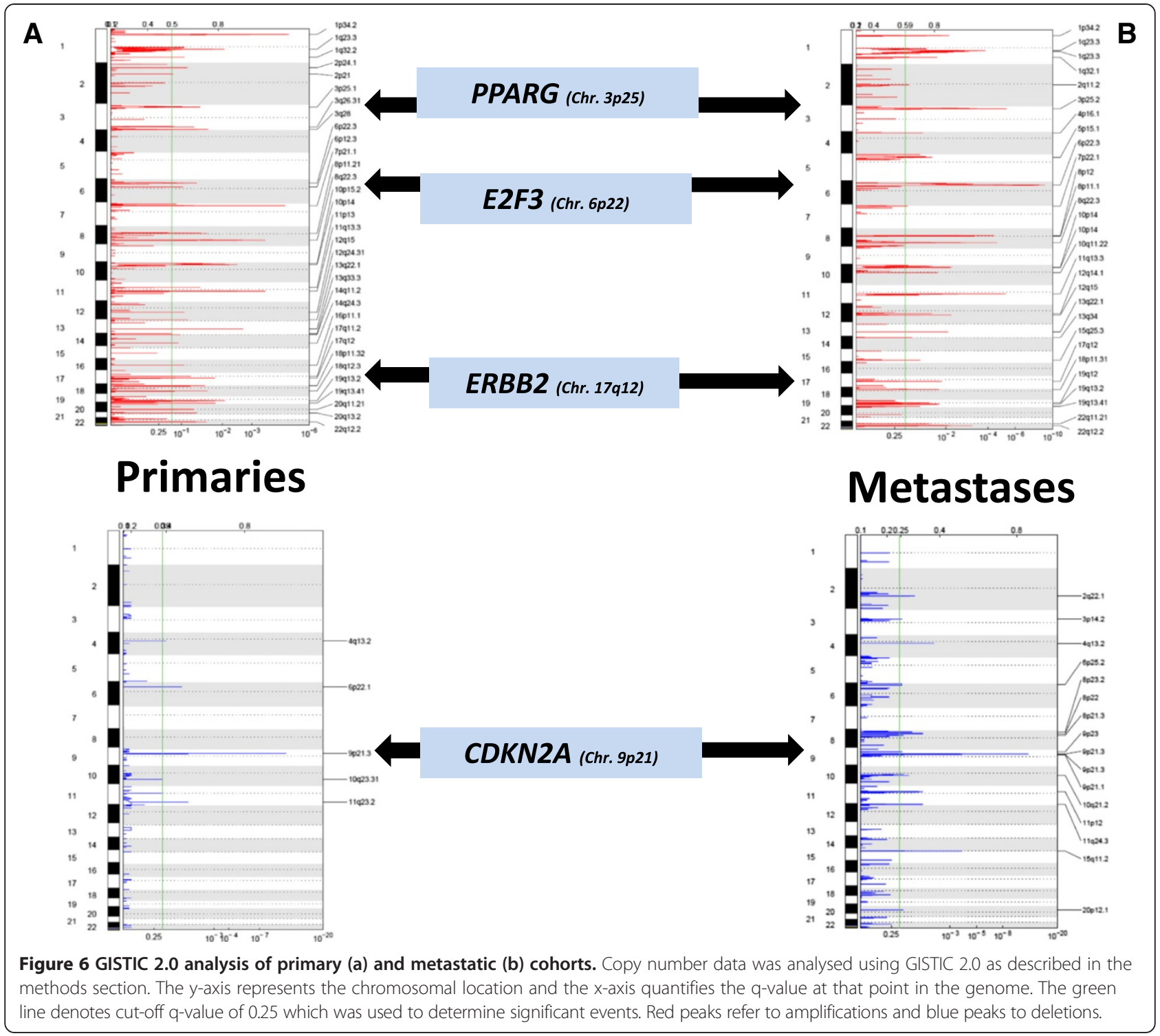

(Figure 7b). 7 of 9 matched primary and metastatic pairs clustered together and for those that did not (patients 160 and 206) the bootstrap values were poor, suggesting that the high confidence pairings (i.e. those with bootstrap values closer to $100 \%$ ) are indeed clonal and that RNA expression profiles globally are maintained between the paired primary and metastatic tumors.

\section{Discussion}

These data provide the first comprehensive assessment of SCNAs in metastatic UC. Amplification or deletion of genes involved in the RB signalling pathway were seen in $60 \%$ (18 of 30) of metastases, which is of interest given the significant activity of CDK4 inhibitors in other cancers $[26,27]$. The data also show a higher frequency of E2F3 amplifications in metastases compared with primary UC and concordant increased E2F3 RNA expression in patients with $E 2 F 3$ amplifications. Prior data from primary UC and other cancers has suggested E2F3 amplification is associated with higher grade and stage primary tumours $[7,28]$. Whether E2F3 activity is a functional driver of metastatic progression or simply a marker for more aggressive disease is not yet clear. Iyer et al. recently showed E2F3 amplification is associated with increased expression of several downstream targets in UC suggesting that, when present, this amplification event results in biologic alterations in this disease [5]. The SOX4 locus, which is located close to $E 2 F 3$, may also be a biologically relevant gene within this amplicon as it is co-amplified in many of these cases as well as having associated increased RNA expression.

Overall, there were more amplifications/deletions in metastases compared with primary tumours. This is in 

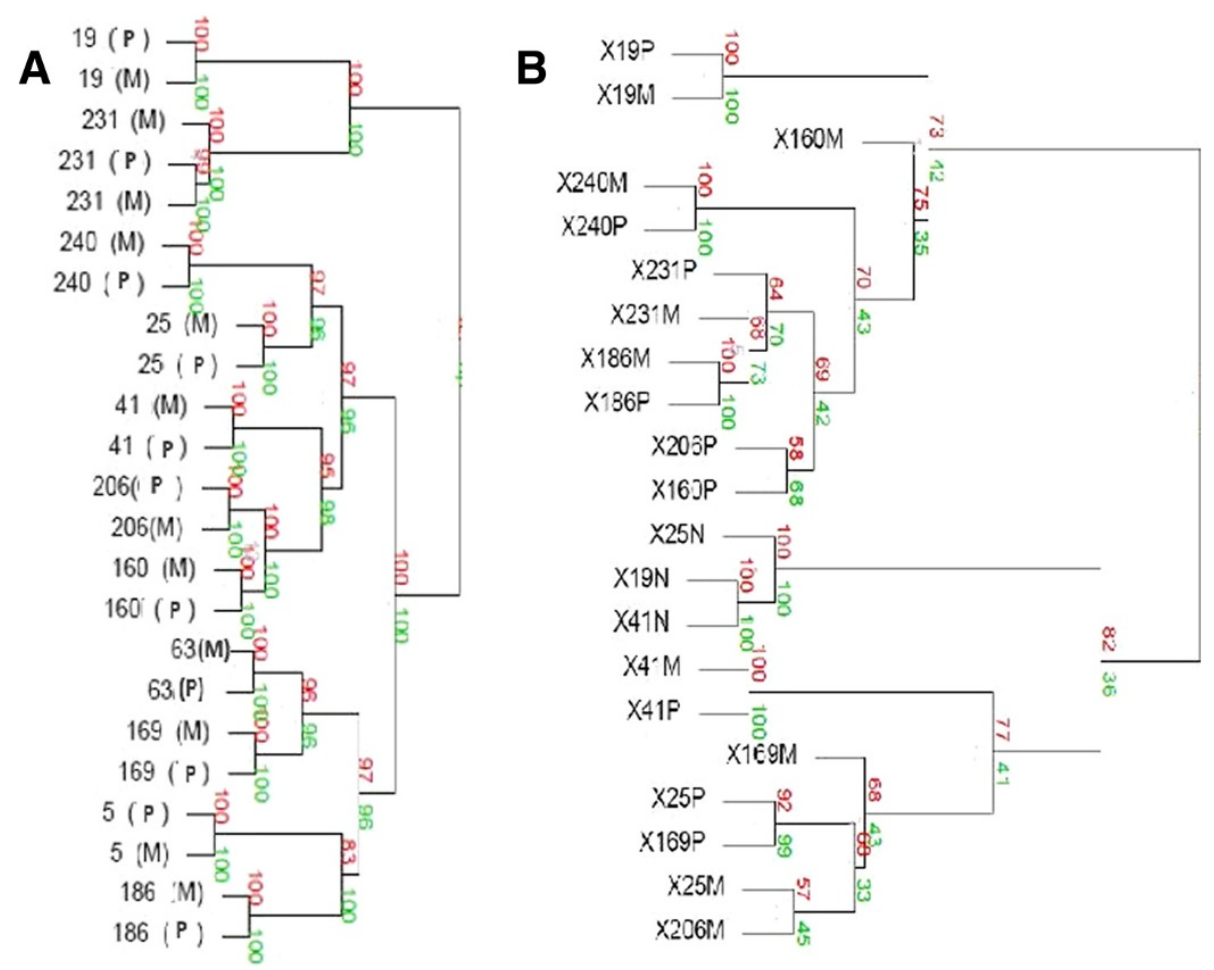

Figure 7 Hierarchical clustering analysis. Hierarchical clustering analysis using both DNA (a) and RNA (b) datasets. The bootstrapping procedure estimates how strongly the clusters are supported by data. Bootstrap values are estimated using multi-scale bootstrap resampling (shown in red numbers) and normal resampling (shown in green numbers), and are reported as percentages, indicating how often a cluster was observed in the bootstrapping [34]. $\mathrm{P}=$ primary tumor, $\mathrm{M}=$ metastasis, $\mathrm{N}$-normal tissue. For the RNA clustering plot, $\mathrm{P}=$ primary tumor, $\mathrm{M}=$ metastasis, $\mathrm{N}=$ normal tissue. Note: for patient 231 two separate brain metastases were analyzed and both clustered together as shown.

keeping with the longstanding model of cumulative genetic change leading to cancer evolution and progression as originally described by Nowell et al. almost 30 years ago [29]. More recently Li et al. demonstrated the clonal evolution of primary bladder UC as illustrated by single cell exome analysis from multiple parts of the same tumor [30]. Of note, there were some instances of amplification in primary tumours that were not present in metastases (e.g. the AHR gene on chromosome 7 in patient 160, Figure 3b) suggesting a divergent rather than longitudinal pattern of evolution whereby different clones can form a branched evolutionary tree despite all arising from a common ancestral cell. This is also in keeping with prior data in this disease [31].

In 3 of 11 patients for whom primary and metastatic tissue was available, there were amplifications in metastases that were not present in the primary tumors, including at the ERBB2, AKT1, CDK4, CCND1 and E2F3 loci. Accompanying total RNA sequencing was available in 2 patients and showed corresponding increased expression levels in several of these genes. This discordance between paired primary and metastatic tissue may have clinical relevance in the era of genomic medicine since the genetic information gleaned from analysing primary tumors may not represent the relevant drivers in metastatic disease. For example, if genomic information from the primary tumour was used to inform therapeutic decision-making for patients 63 and 160 (Figures 3 and 4), the AKT1 and $E R B B 2$ amplifications would not have been evident and these patients would not have been considered for HER2 or AKT-mTOR pathway directed therapies. Studies in colon and lung cancer have found similar instances of discordant SCNAs in cancer-related genes when comparing paired primary and metastatic tissue from the same patients [32,33]. On the other hand, these studies reported high rates of concordance ( $>90 \%$ ) when examining clinically actionable somatic point mutations (including mutations in EGFR and KRAS). The discordance in potentially actionable alterations noted in the data presented here suggest that rates of discordance may differ on a gene-by-gene basis and that discordance in SCNAs may be more common than in somatic point mutations.

One important limitation of the data is the relatively small number of samples analysed which limited the power of the study.

\section{Conclusions}

These data can be used to provide an overview of the SCNA landscape in metastatic UC. The intrapatient 
genomic discrepancies found between primary and metastatic tumours highlights the potential limitations in using archival primary tumour tissue to guide targeted therapy for metastatic disease. Increased frequency of $E 2 F 3$ amplification in metastases points to the relevance of the RB pathway in UC with potential therapeutic implications given the ongoing development of multiple CDK inhibitors.

\section{Additional files}

Additional file 1: Table S1. Sites of tumour and normal tissue used for both DNA and RNA extraction. For each of the 46 patients analysed, the age, gender, tissue site for primary, metastatic and normal control samples are outlined. Data not entered denotes that no specimen was available for that patient. All patients had tumor DNA analysis performed. *denotes patients for which tumor RNA analysis was also performed.

Additional file 2: Figure S1. Comparative marker analysis of differentially expressed genes between normal and tumor tissue. The results of a comparative marker analysis of differentially expressed genes represented as a heat map. Each column denotes a single sample, and each differentially expressed gene is represented in an individual row. Only genes that are differentially expressed in normal bladder (sample number indicated followed by the letter " $N$ ") compared to primaries ("P") and metastases ("M") are displayed. This analysis was performed with 1000 permutations. Red shading indicates higher relative expression and blue shading indicates lower relative expression. In addition to the gene name, a relative rank of the comparative over- or under-expression as well as a p-value and False Discovery Rate (FDR)-corrected and Bonferroni-correct $p$-value are given. The relative fold-change between the aggregate expression in the normal samples versus tumors (primary and metastases) is also presented.

Additional file 3: Figure S2. Comparative marker analysis of differentially expressed genes between primary and metastatic tumor tissue. The results of a comparative marker analysis of differentially expressed genes are presented as a heat map. Each sample is represented in a column and each differentially expressed gene is represented in an individual row. Only genes that are differentially expressed in primaries (" $\mathrm{P}$ ") compared to metastases (" $M$ ") are shown. This analysis was performed with 1000 permutations. Red shading indicates higher relative expression and blue shading indicates lower relative expression. In addition to the gene name, a relative rank of the comparative over- or under-expression as well as a p-value and False Discovery Rate (FDR)-corrected and Bonferronicorrect $p$-value are given. The relative fold-change between the aggregate expression in the primary tumors and metastases is also displayed.

Additional file 4: Figure S3. Low amplitude copy number alterations in matched pairs of primary vs metastatic tumors. Copy number frequency plots for the 11 patients with available matched primary and metastatic tissue. The plots display the frequency of copy number gain (CNG) and copy number loss (CNL) at different points across the genome using a cutoff $\log _{2}$ ratio $+/-0.25$ for $C N G$ and $C N L$, respectively. The $x$ axis represents the different chromosomes and the $y$-axis quantifies the percentage of samples with copy number loss or gain greater than the $+/-0.25 \log _{2}$ ratio cut-off.

Additional file 5: Figure S4. MSKCC clonality tool analysis. Genomic copy-number profiles for all 11 primary tumor/metastasis pairs. These plot visualize copy numbers (log base 2 ratios, $y$-axis) for all segments along the genome (x-axis). Shown odds ratios are calculated using the Clonality $\mathrm{R}$ package. This package provides implementations of statistical tests to determine whether two samples from the same patient are independent or clonal.

\section{Competing interests}

The authors declare that they have no competing interests.

\section{Authors' contributions}

RB was involved in data analysis, interpretation and drafted the manuscript. $A B$ was involved in RNA seq studies, data analysis and helped draft the manuscript. MR was involved in data analysis, interpretation and drafting of the manuscript. CP was involved in RNA seq studies, data analysis and helped draft the manuscript. FD was involved in DNA copy number studies and helped draft the manuscript. JB was involved in DNA copy number studies and helped draft the manuscript. ES was involved in DNA copy number studies and helped draft the manuscript. LW was involved in DNA copy number studies and helped draft the manuscript. RP was involved in DNA copy number studies and helped draft the manuscript. Gl was involved in data analysis, interpretation and helped draft the manuscript. ML was involved in DNA copy number studies and helped draft the manuscript. PK was involved in DNA copy number studies and helped draft the manuscript. FM was involved in DNA copy number studies and helped draft the manuscript. MM was involved in RNA seq studies, data analysis and helped draft the manuscript. JER conceived of the study, and participated in its design and coordination and helped to draft the manuscript. All authors read and approved the final manuscript.

\section{Author details}

${ }^{1}$ Memorial Sloan Kettering Cancer Center/Weill Cornell Medical College, New York, USA. Dana-Farber Cancer Institute/Harvard Medical School, Boston, MA, USA. ${ }^{3}$ The Broad Institute of MIT and Harvard, Cambridge, MA, USA.

Received: 4 August 2014 Accepted: 16 March 2015

Published online: 09 April 2015

\section{References}

1. Jemal A, Bray F, Center MM, Ferlay J, Ward E, Forman D. Global cancer statistics. CA Cancer J Clin. 2011;61(2):69-90.

2. Eble JN, World Health Organization Classification of Tumours, Sauter G, Epstein JI, Sesterhenn IA. Pathology and Genetics of Tumours of the Urinary System and Male Genital Organs. Lyon, France: IARC Press; 2004.

3. The Cancer Genome Atlas Research N. Comprehensive molecular characterization of urothelial bladder carcinoma. Nature. 2014;507(7492):315-22

4. Kompier LC, Lurkin I, van der Aa MN, van Rhijn BW, van der Kwast TH, Zwarthoff EC. FGFR3, HRAS, KRAS, NRAS and PIK3CA mutations in bladder cancer and their potential as biomarkers for surveillance and therapy. PLoS One. 2010;5(11):e13821.

5. Iyer G, Al-Ahmadie H, Schultz N, Hanrahan AJ, Ostrovnaya I, Balar AV, et al. Prevalence and Co-occurrence of actionable genomic alterations in high-grade bladder cancer. J Clin Oncol Off J Am Soc Clin Oncol. 2013;31(25):3133-40.

6. Gui Y, Guo G, Huang Y, Hu X, Tang A, Gao S, et al. Frequent mutations of chromatin remodeling genes in transitional cell carcinoma of the bladder. Nat Genet. 2011:43(9):875-8.

7. Lindgren D, Sjodahl G, Lauss M, Staaf J, Chebil G, Lovgren K, et al. Integrated genomic and gene expression profiling identifies two major genomic circuits in urothelial carcinoma. PLoS One. 2012;7(6):e38863.

8. Hurst CD, Platt FM, Taylor CF, Knowles MA. Novel tumor subgroups of urothelial carcinoma of the bladder defined by integrated genomic analysis. Clin Cancer Res. 2012;18(21):5865-77.

9. Ross JS, Wang K, Al-Rohil RN, Nazeer T, Sheehan CE, Otto GA, et al. Advanced urothelial carcinoma: next-generation sequencing reveals diverse genomic alterations and targets of therapy. Mod Pathol 20132014 27(2):271-80.

10. Qiagen. QIAamp DNA FFPE Tissue Handbook. 2010

11. Wang Y, Carlton VE, Karlin-Neumann G, Sapolsky R, Zhang L, Moorhead $M$, et al. High quality copy number and genotype data from FFPE samples using Molecular Inversion Probe (MIP) microarrays. BMC Med Genet. 2009;2:8

12. Darvishi K. Application of Nexus copy number software for CNV detection and analysis. Current protocols in human genetics/editorial board, Jonathan L Haines [et al.]. 2010;Chapter 4:Unit 4 14 1-28.

13. Levin JZ, Berger MF, Adiconis X, Rogov P, Melnikov A, Fennell T, et al. Targeted next-generation sequencing of a cancer transcriptome enhances detection of sequence variants and novel fusion transcripts. Genome Biol. 2009;10(10):R115. 
14. Bentley DR, Balasubramanian S, Swerdlow HP, Smith GP, Milton J, Brown $C G$, et al. Accurate whole human genome sequencing using reversible terminator chemistry. Nature. 2008:456(7218):53-9.

15. Berger MF, Levin JZ, Vijayendran K, Sivachenko A, Adiconis X, Maguire J, et al. Integrative analysis of the melanoma transcriptome. Genome Res. 2010;20(4):413-27.

16. Kostic AD, Ojesina Al, Pedamallu CS, Jung J, Verhaak RG, Getz G, et al. PathSeq: software to identify or discover microbes by deep sequencing of human tissue. Nat Biotechnol. 2011;29(5):393-6.

17. DeLuca DS, Levin JZ, Sivachenko A, Fennell T, Nazaire MD, Williams C, et al. RNA-SeQC: RNA-seq metrics for quality control and process optimization. Bioinformatics. 2012;28(11):1530-2.

18. Lockwood WW, Chari R, Coe BP, Girard L, Macaulay C, Lam S, et al. DNA amplification is a ubiquitous mechanism of oncogene activation in lung and other cancers. Oncogene. 2008;27(33):4615-24.

19. Thu KL, Radulovich N, Becker-Santos DD, Pikor LA, Pusic A, Lockwood WW, et al. SOX15 is a candidate tumor suppressor in pancreatic cancer with a potential role in Wnt/beta-catenin signaling. Oncogene. 2013 2014;33(3):279-88.

20. Hupe P, Stransky N, Thiery JP, Radvanyi F, Barillot E. Analysis of array CGH data: from signal ratio to gain and loss of DNA regions. Bioinformatics. 2004;20(18):3413-22.

21. Mermel $\mathrm{CH}$, Schumacher SE, Hill B, Meyerson ML, Beroukhim R, Getz G. GISTIC2.0 facilitates sensitive and confident localization of the targets of focal somatic copy-number alteration in human cancers. Genome Biol. 2011;12(4):R41

22. Ostrovnaya I, Olshen AB, Seshan VE, Orlow I, Albertson DG, Begg CB. A metastasis or a second independent cancer? Evaluating the clonal origin of tumors using array copy number data. Stat Med. 2010;29(15):1608-21.

23. Ostrovnaya I, Seshan VE, Begg CB. Comparison of properties of tests for assessing tumor clonality. Biometrics. 2008;64(4):1018-22.

24. Begg CB, Eng KH, Hummer AJ. Statistical tests for clonality. Biometrics. 2007;63(2):522-30

25. Riester M, Werner L, Bellmunt J, Selvarajah S, Guancial EA, Weir BA, et al. Integrative analysis of 1q23.3 copy number gain in metastatic urothelial carcinoma. Clin Cancer Res. 2014;20(7):1873-83

26. Dickson MA, Tap WD, Keohan ML, D'Angelo SP, Gounder MM, Antonescu CR, et al. Phase II Trial of the CDK4 Inhibitor PD0332991 in Patients With Advanced CDK4-Amplified Well-Differentiated or Dedifferentiated Liposarcoma. J Clin Oncol. 2013:31(16):2024-8.

27. Finn RS, Crown JP, Lang I, Boer K, Bondarenko IM, Kulyk SO, et al., editors. Results of a randomized phase 2 study of PD 0332991, a cyclin dependent kinase (CDK) 4/6 inhibitor, in combination with letrozole vs letrozole alone for first-line treatment of ER+/HER2- advanced breast cancer (BC). San Antonio Breast Cancer Symposium 2012; 2012; San Antonio, Texas, USA.

28. Beroukhim R, Mermel CH, Porter D, Wei G, Raychaudhuri S, Donovan J, et al. The landscape of somatic copy-number alteration across human cancers. Nature. 2010;463(7283):899-905

29. Nowell PC. The clonal evolution of tumor cell populations. Science. 1976;194(4260):23-8

30. Li Y, Xu X, Song L, Hou Y, Li Z, Tsang S, et al. Single-cell sequencing analysis characterizes common and cell-lineage-specific mutations in a muscle-invasive bladder cancer. GigaScience. 2012;1(1):12.

31. Cha EK, Sfakianos JP, Al-Ahmadie H, Scott SN, Kim PH, lyer G, et al. Branched evolution and intratumor heterogeneity of urothelial carcinoma of the bladder. ASCO Meeting Abstracts. 2014;32(4_suppl):293.

32. Vakiani $E$, Janakiraman $M$, Shen $R$, Sinha R, Zeng Z, Shia J, et al. Comparative genomic analysis of primary versus metastatic colorectal carcinomas. J Clin Oncol Off J Am Soc Clin Oncol. 2012;30(24):2956-62.

33. Vignot S, Frampton GM, Soria JC, Yelensky R, Commo F, Brambilla C, et al. Nextgeneration sequencing reveals high concordance of recurrent somatic alterations between primary tumor and metastases from patients with non-small-cell lung cancer. J Clin Oncol Off J Am Soc Clin Oncol. 2013;31(17):2167-72.

34. Shimodaira $\mathrm{H}$. Approximately unbiased tests of regions using multistepmultiscale bootstrap resampling. Ann Stat. 2004:32:2616-41.

\section{Submit your next manuscript to BioMed Central and take full advantage of:}

- Convenient online submission

- Thorough peer review

- No space constraints or color figure charges

- Immediate publication on acceptance

- Inclusion in PubMed, CAS, Scopus and Google Scholar

- Research which is freely available for redistribution

Submit your manuscript at www.biomedcentral.com/submit 